\title{
Large (14.5 dB) reduction of intensity noise from a semiconductor laser by amplitude-phase decorrelation
}

\author{
Michael A. Newkirk ${ }^{\text {a) }}$ and Kerry J. Vahala \\ Department of Applied Physics, 128-95, California Institute of Technology, Pasadena, California 91125
}

(Received 30 October 1991; accepted for publication 3 January 1992)

\begin{abstract}
By the method of amplitude-phase decorrelation, intensity noise from a distributed feedback semiconductor laser is passively reduced up to $14.5 \mathrm{~dB}$ below its intrinsic level. At high laser power, reduction to $1.3 \mathrm{~dB}$ above the photon shot noise level is observed. The results agree with a simple model incorporating a power-independent source of phase noise in the laser rate equations.
\end{abstract}

The fundamental coupling between field amplitude and phase fluctuations in a semiconductor laser is measured by the $\alpha$ parameter. ${ }^{1,2}$ This coupling is not only responsible for the well-known enhancement of the fundamental linewidth by a factor of $1+\alpha^{2}$ over the Schawlow-Townes linewidth, but also induces correlation between the fluctuations. Recently we demonstratcd that this correlation may be used to reduce intensity noise by a method called amplitude-phase decorrelation. ${ }^{3,4}$ The method employs a frequency-dependent transmission function, such as a Michelson interferometer, to convert phase noise to intensity noise. We showed theoretically that reduction by the factor $1 /\left(1+\alpha^{2}\right)$ is possible, and experimentally observed up to a factor of $5(7 \mathrm{~dB})$ decrease in the intensity noise level of a distributed feedback (DFB) semiconductor laser.

In this letter, we investigate noise reduction by amplitude-phase decorrelation in relation to the photon shot noise level (SNL). The SNL represents a classical lower bound for intensity noise, and the goal of reduction below the SNL (or standard quantum limit) has motivated much research on the generation of sub-Poissonian (squeezed) light. $^{5-9}$ Using a balanced homodyne detector, both the laser intensity noise level and the SNL can be measured. For a DFB laser at low power-in the excess noise regime-we observe intensity noise reduction by a factor of $28(14.5 \mathrm{~dB})$, but the reduced noise level is $9 \mathrm{~dB}$ above the SNL. At high power where the intrinsic noise level is closer to the SNL, reduction to $1.3 \mathrm{~dB}$ above the SNL is observed, but the amount of reduction is decreased. We show that inhibition of intensity noise reduction at high power agrees with a simple model incorporating a power-independent source of lasing-mode phase noise.

The laser field fluctuations are represented semiclassically by the slowly varying phasor $A(t)=A_{0}[1$ $+\rho(t)] \exp [i \varphi(t)]$, with small-signal amplitude and phase fluctuations $\rho$ and $\varphi$. In the frequency domain (where we only consider fluctuation frequencies $\Omega \ll$ the population damping rate, typically several $\mathrm{GHz}$ for a semiconductor laser) rate equations for the fluctuations are given by ${ }^{3,4}$

$$
\begin{aligned}
& \rho(\Omega)=\frac{\Delta_{R}}{\tau_{r} \omega_{r}^{2}}, \\
& \dot{\varphi}(\Omega)=\Delta_{I}+\alpha \Delta_{R}+\Delta_{0},
\end{aligned}
$$

\footnotetext{
^) Present address: AT\&T Bell Laboratories, Holmdel, NJ 07733.
}

where the variable $\dot{\varphi}(\Omega)$ is the Fourier transform of $\dot{\varphi}(t)$, the instantaneous frequency fluctuation. $\Delta_{R}$ and $\Delta_{I}$ are the real and imaginary parts of the Langevin noise source accounting for spontaneous emission into the lasing mode, and $\omega_{r}$ and $\tau_{r}$ are the frequency and damping time of the relaxation oscillations. As seen above, the $\alpha$ parameter measures the coupling of amplitude noise into excess phase noise. $\Delta_{R}$ and $\Delta_{I}$ are uncorrelated and have a flat spectral density given by

$$
\left\langle\Delta_{R}^{2}\right\rangle=\left\langle\Delta_{I}^{2}\right\rangle=\frac{S}{2 P}
$$

where $S$ is the average rate of spontaneous emission into the lasing mode, $P$ is the average photon number in the mode, and $S / 2 P \equiv \Delta \omega_{\mathrm{ST}}$ is the modified Schawlow-Townes linewidth. In Eq. (1b), $\Delta_{0}$ is an independent phase noise source, uncorrelated with $\Delta_{R}$ and $\Delta_{I}$, which models the origin of a phenomenological linewidth floor occurring in semiconductor lasers. [Note: a linewidth floor exists in all DFB and distributed Bragg reflector lasers, but its cause is unknown]. ${ }^{10}$ The spectral density $\left\langle\Delta_{0}^{2}\right\rangle \equiv \Delta \omega_{0}$ is flat but independent of laser power, so that the laser linewidth, given by $\left(1+\alpha^{2}\right) \Delta \omega_{\mathrm{ST}}+\Delta \omega_{0}$ (the spectral density of $\left.\dot{\varphi}\right)$, varies inversely with increasing laser power and eventually saturates to the value $\Delta \omega_{0}$. Aside from giving rise to a linewidth floor, $\Delta_{0}$ has the deleterious effect of diluting the correlation between $\rho$ and $\dot{\varphi}$.

The method of amplitude-phase decorrelation consists of passing the laser field through a dispersive transmission function, such as a Michelson interferometer, and then using the correlation between the fluctuations to reduce the level of intensity noise. The Michelson, detuned from maximum transmission, acts as a frequency discriminator, i.e., the amplitude transmission varies with frequency. Hence, instantaneous frequency noise at the input is converted to amplitude noise. The output amplitude fluctuation is thus a mixture of the input fluctuations

$$
\rho_{0}(\Omega)=\rho_{i}(\Omega)+\xi \dot{\varphi}_{i}(\Omega),
$$

where

$$
\xi=\frac{1}{T_{R}} \frac{d}{d \omega} T_{R}
$$

is the normalized slope of the real part of the transmission function, evaluated at the lasing frequency. ${ }^{4}$ Relative inten- 


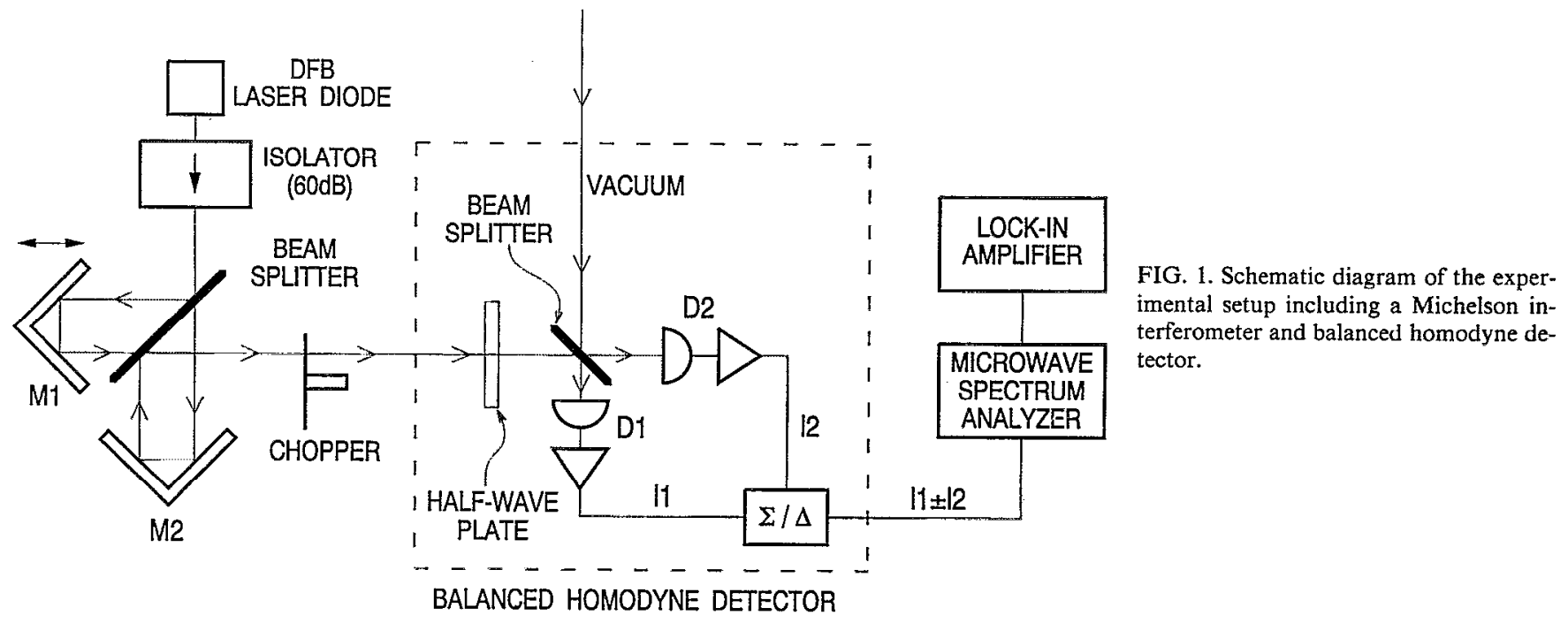

sity noise after the Michelson, proportional to the spectral density of $\rho_{0}$, is given by

$$
\mathrm{RIN}_{0} \propto\left\langle\rho_{0}^{2}\right\rangle=\left\langle\rho_{i}^{2}\right\rangle+\xi^{2}\left\langle\dot{\varphi}_{i}^{2}\right\rangle+2 \xi\left\langle\rho_{i} \dot{\varphi}_{i}\right\rangle,
$$

which is governed by the slope $\xi$. Minimizing with respect to $\xi$ gives the maximum reduction

$$
\frac{\mathrm{RIN}_{0}}{\operatorname{RIN}_{i}}=\frac{\left\langle\rho_{0}^{2}\right\rangle}{\left\langle\rho_{i}^{2}\right\rangle}=1-\frac{\left\langle\rho_{i} \dot{\varphi}_{i}\right\rangle^{2}}{\left\langle\rho_{i}^{2}\right\rangle\left\langle\dot{\varphi}_{i}^{2}\right\rangle},
$$

where the initial correlation $\left\langle\rho_{i} \dot{\varphi}_{i}\right\rangle$ makes noise reduction possible. One can also show that for the field fluctuations after the Michelson, $\left\langle\rho_{0} \dot{\varphi}_{0}\right\rangle=0$, hence the name amplitude-phase decorrelation. Using the above spectral densities for the noise sources, the maximum reduction can be expressed as

$$
\frac{\operatorname{RIN}_{0}}{\operatorname{RIN}_{i}}=\frac{1+\beta}{1+\alpha^{2}+\beta},
$$

where $\beta \equiv \Delta \omega_{0} / \Delta \omega_{\mathrm{ST}}$ is the ratio of the spectral density of $\Delta_{0}$ to the Schawlow-Townes linewidth. Hence, for an ideal laser with no linewidth floor, i.e., $\Delta \omega_{0}=0$, a maximum reduction by $1 /\left(1+\alpha^{2}\right)$, independent of laser power, is possible. When $\Delta \omega_{0}$ is nonzero, however, the reduction is power dependent because $\beta$ increases linearly with laser power.

The experimental arrangement appears in Fig. 1. The output from a $1.3 \mu \mathrm{m} \mathrm{InGaAs/InGaAsP} \mathrm{DFB} \mathrm{laser} \mathrm{is} \mathrm{sent}$ through a $60 \mathrm{~dB}$ optical isolator to a Michelson interferometer. The slope of the Michelson transmission function is controlled by adjusting the optical path difference of the two arms using a micrometer and then detuning from maximum transmission using a piezoelectric transducer. Path differences of a few $\mathrm{mm}$ provide optimum slopes near halfintensity transmission. After the Michelson, the light goes to a balanced homodyne detector (BHD), where it is divided by a half-wave plate and polarization-sensitive beam splitter. (The physical principles of balanced detection are discussed in Refs. 11 and 12.) Two matched InGaAs/InP $p-i-n$ photodiodes (BT\&D PDH0004) with 0.90 quantum efficiency are used with a bias tce and high-gain, low-noise amplifier (Avantek ACT10-213-1). The amplified ac currents are combined in a broadband hybrid junction which may coherently add or subtract the two inputs. The combined noise photocurrents are sent to a microwave spectrum analyzer to measure noise power. Lock-in detection is also used to improve the sensitivity and filter thermal noise.

The optical powers in the two detector arms are balanced by rotating the half-wave plate. It is also important that the path lengths are equal, accomplished by current modulating the DFB at the measurement frequency, subtracting the photocurrents with the hybrid junction, and adjusting the detector differential path length until the modulation peak seen on the spectrum analyzer is minimized. By this approach, a common mode rejection of 60 $\mathrm{dB}$ could be obtained. Once the BHD is balanced, the laser's intensity noise level or the SNL is observed by switching between the summing and differencing output ports of the hybrid junction, respectively. The performance of the BHD was verified by inserting neutral density filters between the laser and detector and observing the correct variation of the measured SNL as a function of attenuation to within $0.1 \mathrm{~dB}^{7}$

Noise measurements were performed on the DFB laser for a range of bias currents from 15.5 (1.07 times threshold current) up to $40.0 \mathrm{~mA}$. Figure 2 shows noise power in terms of RIN as a function of average laser power after the Michelson. For each bias current the shot noise level, intrinsic noise level, and maximally reduced noise level are shown. Noise power was measured in a $100 \mathrm{kHz}$ bandwidth at $17.8 \mathrm{MHz}$, an arbitrary frequency in the flat region of the intensity noise spectrum; other measurement frequencies showed similar results. The Michelson was held at half-intensity transmission for each measurement, and the transmission slope was adjusted as discussed above. We see that at $15.5 \mathrm{~mA}$ bias $(-12 \mathrm{dBm}$ power $)$ noise can be reduced by a factor of $28.6(14.5 \mathrm{~dB})$ below the intrinsic level. As the bias increases, the intrinsic noise level falls and the amount of reduction decreases as the reduced noise approaches the SNL. At $40.0 \mathrm{~mA}$ bias (3.7 


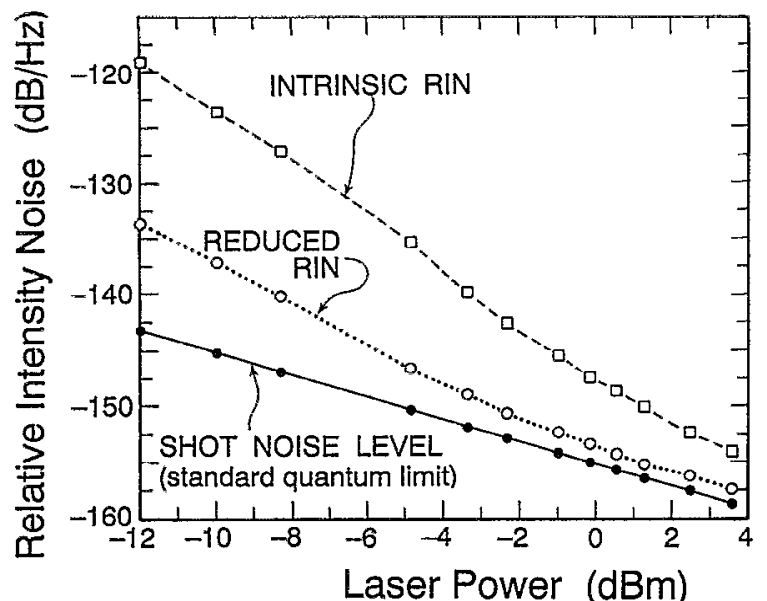

FIG. 2. Intrinsic RIN, reduced RIN, and the shot noise level vs laser power after the Michelson. Laser bias currents range from 15.5 to 40.0 $\mathrm{mA}$. Intensity noise can be reduced by $14.5 \mathrm{~dB}$ at low bias. At high bias, the noise is reduced to $1.3 \mathrm{~dB}$ above the $\mathrm{SNL}$, but the amount of reduction is decreased.

$\mathrm{dBm}$ power) the noise level can only be reduced $3.4 \mathrm{~dB}$ below the intrinsic level, and the reduced noise is $1.3 \mathrm{~dB}$ above the SNL.

The observed power dependence of the noise reduction is in agreement with Eq. (6), where the presence of the $\beta$ parameter inhibits noise reduction at high power. For this laser it was not possible to directly determine $\beta$ from a linewidth measurement, because the laser could not be operated at high enough power to observe linewidth saturation, which would reveal $\Delta \omega_{0}$ (we have observed highpower linewidth saturation in other DFB lasers). Nevertheless, rewriting Eq. (6) as

$$
\beta=\frac{\alpha^{2}}{\left(\operatorname{RIN}_{i} / \operatorname{RIN}_{0}\right)-1}-1,
$$

the power dependence of $\beta$ can be extracted from the data in Fig. 2. In Fig. 3 we plot $\beta$ as a function of RIN $_{0} /$ RIN $_{i}$ vs laser power. $|\alpha|$ is taken to be 5.25 , consistent with the factor of 28.6 reduction near threshold where $\beta$ is negligible. We see that $\beta$ vs laser power fits well to a line, in agreement with the definition of $\beta$ [below Eq. (6)] and the simple model incorporating a power-independent phase noise source in the rate equations.

Although the semiclassical formulation of amplitudephase decorrelation, used in the letter, agrees with the experimental results, the issue of noise reduction near the SNL properly requires a fully quantum-mechanical treatment of the field. The impact of noise sources such as the vacuum field entering the Michelson's open port can then

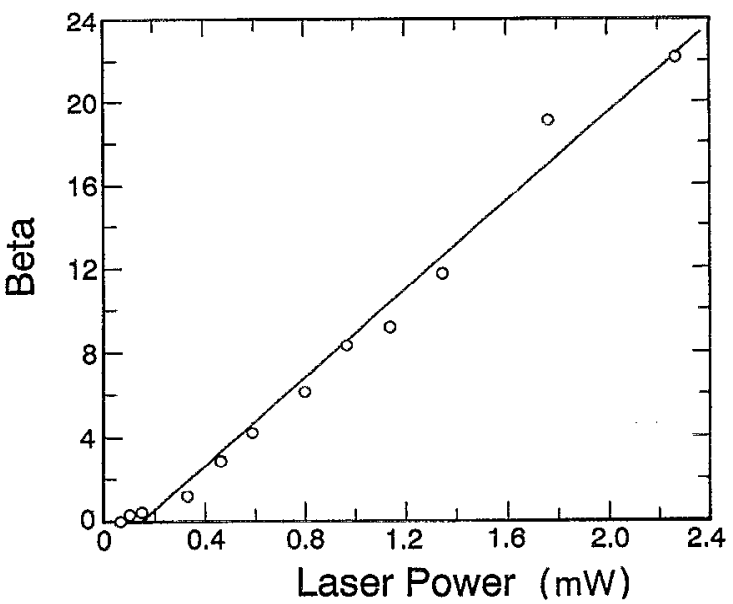

FIG. 3. Beta, from Eq. (7), vs laser power. A linear relationship, consistent with the presence of a power-independent source of phase noise in the rate equations, is observed.

easily be evaluated, but the essential features of the technique should be preserved.

In conclusion, the method of amplitude-phase decorrelation was used to passively reduce intensity noise from a distributed feedback semiconductor laser by as much as a factor of 28 below its intrinsic level. Using a balanced homodyne detector, noise reduction as a function of laser power was measured relative to the photon shot noise level. At high power, the decorrelated noise could be reduced to $1.3 \mathrm{~dB}$ above the SNL. Further reduction appears to be inhibited by a power-independent source of phase noise in the lasing mode.

The authors thank T. R. Chen of Ortel Corporation for the DFB laser used in this experiment. This work is supported by the Office of Naval Research.

${ }^{1}$ C. H. Henry, IEEE J. Quantum Electron. QE-18, 259 (1982).

${ }^{2}$ K. Vahala and A. Yariv, IEEE J. Quantum Electron. QE-19, 1096 (1983).

${ }^{3}$ K. J. Vahala and M. A. Newkirk, Appl. Phys. Lett. 57, 974 (1990).

${ }^{4}$ M. A. Newkirk and K. J. Vahala, IEEE J. Quantum Electron. QE-27, 13 (1991).

${ }^{5}$ L. Wu, H. J. Kimble, J. L. Hall, and H. Wu, Phys. Rev. Lett. 57, 2520 (1986).

${ }^{6}$ R. E. Slusher, L. W. Hollberg, B. Yurke, J. C. Mertz, and J. F. Valley, Phys. Rev. Lett. 55, 2409 (1985).

${ }^{7}$ G. J. Milburn, M. D. Levenson, R. M. Shelby, S. H. Perlmutter, R. G. DeVoe, and D. F. Walls, J. Opt. Soc. Am. B 4, 1476 (1987).

${ }^{8}$ S. Machida, Y. Yamamoto, and Y. Itaya, Phys. Rev. Lett. 58, 1000 (1987).

9 J. Mertz, A. Heidmann, C. Fabre, E. Giacobino, and S. Reynaud, Phys. Rev. Lett. 64, 2897 (1990).

${ }^{10}$ T. L. Koch and U. Koren, J. Lightwave Technol. LT-8, 274 (1990).

${ }^{11}$ H. P. Yuen and V. W. S. Chan, Opt. Lett. 8, 177 (1983).

${ }^{12}$ B. Schumaker, Opt. Lett. 9, 189 (1984). 